\title{
Medial Preoptic Sexual Dimorphisms in the Guinea Pig. I. An Investigation of Their Hormonal Dependence
}

\author{
William Byne and Ruth Bleier \\ Neurosciences Training Program and Department of Neurophysiology, University of Wisconsin, Madison, Wisconsin 53706
}

\begin{abstract}
The guinea pig exhibits sexually dimorphic patterns of cell density and distribution throughout the medial preoptic area, a region that has been shown to be involved in the regulation of sexually differentiated behavioral and endocrinological reproductive functions (Bleier et al., 1982). The most prominent sex differences involve 2 components of the medial preoptic nucleus (MPN), an anteriorly placed compact subnucleus (MPNa) that is twice as large in females as in males, and a centrally placed compact subnucleus that occupies an approximately 10 -fold greater volume in males than in females and corresponds to the sexually dimorphic nucleus described in the rat by Gorski et al. (1978). In the present study the sex differences in both of these cell groups were shown to be unaffected by neonatal gonadectomy and postnatal hormonal manipulations. In contrast, MPNa volume was significantly decreased and MPNC volume significantly increased in genotypic females exposed to testosterone propionate (TP) on gestational days 28-37 or 28-65 but not 3865. All 3 prenatal TP treatments administered to females significantly increased mounting frequencies and suppressed lordosis, ovulation, and the positive-feedback effects of estrogen and progesterone on luteinizing hormone release. Thus, the volumetric sex differences in MPNa and MPNc alone do not seem to be sufficient to account for the sex differences in the functions of the medial preoptic region. It is, therefore, suggested that androgens continue to exert organizational influences upon the developing brain after cytoarchitectonic patterns have been determined.
\end{abstract}

During the past decade and a half, neuroanatomical sexual dimorphisms of various types have been described in the medial preoptic-anterior hypothalamic region (MPAH) of several laboratory rodents. The significance of these findings lies in the fact that the MPAH participates in the regulation of a number of behavioral and neuroendocrinological functions that, in rodents, become sexually differentiated in response to sex differences in exposure to androgens or their metabolites in early develop-

\footnotetext{
Received Sept. 23, 1986; revised Feb. 23, 1987; accepted Feb. 26, 1987.

W.B. was a predoctoral fellow in the Neurosciences Training Program at the University of Wisconsin at the time of this study. We are very grateful to Inge Siggelkow, Joan Meister, and Jo Ann Ekleberry for their careful and skillful histological work, to John Warren for his technical assistance, and to Robert Goy, Ei Terasawa, and Melissa Hines for their suggestions and careful reading of the original manuscript. This work has been supported by grants from the NIH (NS16643 and HDO3352) and the University of Wisconsin Graduate School and by a neurosciences training grant (NIH GM-07507).

Correspondence should be addressed to William Byne, Albert Einstein College of Medicine, Box 52, 1300 Morris Park Avenue, Bronx, NY 10461.

Copyright (C) 1987 Society for Neuroscience $0270-6474 / 87 / 092688-09 \$ 02.00 / 0$
}

ment. The neuroanatomical sex differences are, therefore, thought to be the signature of the organizational action of androgens on sexually differentiated brain functions. Electron microscopic studies have revealed sex differences in the synaptic organization of the strial portion of the preoptic area of the rat (Raisman and Field, 1973), and in Golgi-stained material sex differences in dendritic branching patterns have been described in the preoptic area of the hamster (Greenough et al., 1977) and rat (Meyers and Gordon, 1982). Also in the rat, electrophysiological studies have demonstrated sex differences in the convergence of synaptic inputs to the preoptic area and anterior hypothalamus (Dyer et al., 1976). In addition to these subtle sexual dimorphisms, conspicuous sexual dimorphisms have been described in the cytoarchitecture of nuclei within the MPAH of the rat, hamster, mouse, gerbil, ferret, and guinea pig (Gorski et al., 1978, 1980; Bleier et al., 1982; Commins and Yahr, 1984a; Hines et al., 1985; Tobet et al., 1986).

The sexually dimorphic nuclei within the MPAH bind high levels of gonadal steroids during the period within which steroidal manipulations influence the sexual differentiation of brain function (Sar and Stumpf, 1975a, b; Sheridan et al., 1975; Vito et al., 1979), and several of the dimorphisms have been shown to develop in response to sex differences in steroidal exposure during this same period (Raisman and Field, 1973; Greenough et al., 1977; Gorski et al., 1978). The sexual dimorphism in the gerbil brain, however, has been shown to be influenced by circulating steroids in adulthood (Commins and Yahr, 1984a, b).

In a previous report we described a complex of cytoarchitectonic sex differences within the MPAH of the guinea pig (Bleier et al., 1982). Because the dimorphisms involved the boundaries and ccllular groupings within the entire region, we called the dimorphic region the "sexually dimorphic medial preoptic-anterior hypothalamic continuum." The most prominent dimorphisms involve an anterior portion of the continuum that we called the medial preoptic nucleus (MPN) and a more posteriorly situated portion that we called the anterior hypothalamic nucleus (AH). The MPN was described as being more prominent in females than males, while $\mathrm{AH}$ in the male was reported to contain a dorsally placed subgroup of increased density corresponding to the sexually dimorphic nucleus of the preoptic area (SDN-POA) in the rat described by Gorski et al. (1978). In contrast, $\mathrm{AH}$ in the female was reported to be almost uniformly cellular throughout. In the present study we describe the quantitative parameters of the cytoarchitectonic sexual dimorphisms involving these nuclei and the nature of their hormonal dependence. The guinea pig was chosen for these studies because it may provide a better model than other rodents of particular developmental and neuroendocrine processes in primates. Un- 
like other laboratory rodents, the major events of its sexual differentiation occur prenatally as in the primate, and in some ways it is more similar to the primate with respect to the organization and function of the hypothalamic-pituitary-gonadal axis. Specifically, unlike the rat, its ovarian cycle includes a true luteal phase and its medial basal hypothalamus is capable of supporting ovarian cyclicity following complete deafferentation (Butler and Donovan, 1970; Terasawa and Wiegand, 1977; Terasawa et al., 1984).

In light of the troublesome differences that have arisen involving the naming of medial preoptic cell groups, some comments on nomenclature are in order. In our interpretation the nuclei that we have previously referred to as MPN and AH were designated, respectively, as medial preoptic nuclei $b$ and $a$ by Gurdjian (1927). It should be noted that there are several ambiguities or contradictions in Gurdjian's description of the medial preoptic area and that nuclei $a$ and $b$ are not labeled in his illustrations. Because Gurdjian (pp. 79, 81) relied on the plane of the cephalic extreme of the nucleus ovoidus (suprachiasmatic nucleus) to delineate the cell group labeled "medial preoptic nucleus" in his figure 1 from the posterior continuation of that same cell group labeled "anterior hypothalamic nucleus" in his figure 2 , he arbitrarily assigned 2 names to the rostral and caudal portions of a single cytoarchitectonic configuration. For this reason we have previously referred to that configuration as the anterior hypothalamic nucleus and retained the designation of medial preoptic nucleus for what according to our interpretation is the more rostrally placed medial preoptic nucleus $b$ of Gurdjian. Following the nomenclature of Konig and Klippel (1963), which also attempts to follow Gurdjian, most authors have referred to the entire medial preoptic area (MPA), including Gurdjian's medial preoptic nucleus $a$ (our AH), as the medial preoptic nucleus.

In an attempt to avoid the ambiguities that have arisen from differences in the interpretation of Gurdjian's work, we have allered our nomenclature as follows: MPN and AH of the previous study together with the transitional zone between them are considered to constitute the medial preoptic nucleus (MPN), and we will refer to the MPN of the previous study as the anterior compact subnucleus (MPNa). The cell group corresponding to the SDN-POA of Gorski et al. will be referred to as the central compact subnucleus (MPNc), and the more moderately cellular portions of the MPN will be referred to as the principal part of the nucleus (MPNp). These changes follow the recommendations of S. J. Wiegand, R. E. Watson, and G. E. Hoffman (personal communication) based on their recent detailed immunohistochemical mapping studies. While their findings are compatible with our previous parcellation of the "medial preoptic-anterior hypothalamic continuum" and our concepts regarding the organization of this continuum as a "sexually dimorphic nuclear complex" (Bleier et al., 1982), they prefer to extend the region designated as the medial preoptic area so that it includes all of the involved nuclei. The schema of Wiegand et al. has the advantage of retaining our scheme of parcellation while making the nomenclature more compatible with that in common usage and that adopted by Paxinos and Watson (1986) in the second edition of their stereotaxic atlas of the rat brain. Our previous nomenclature is compared with that of the present study in Figure 1. It should also be noted that the MPNa of the present study has been referred to as the anteroventral periventricular nucleus in 2 recent studies involving rats (Simerly et al., $1985 \mathrm{a}, \mathrm{b})$.
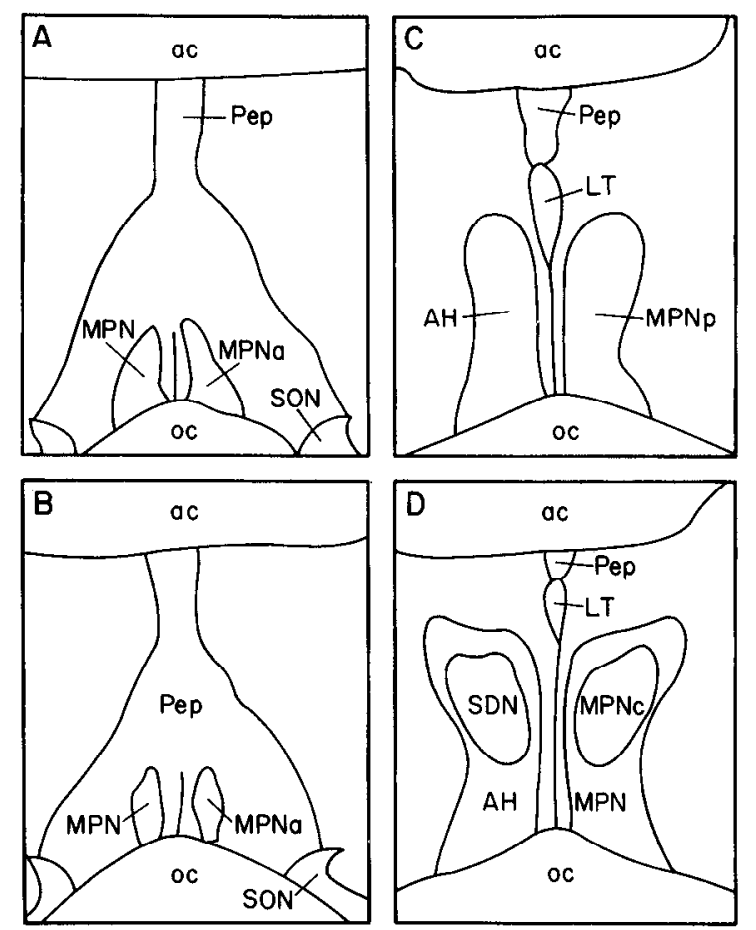

Figure 1. Comparison of our previous with our present nomenclature. Our previous nomenclature is indicated on the left side of each illustration and our present nomenclature is indicated on the right. $A$ (Female) and $B$ (Male) are through the anterior portion of the medial preoptic area corresponding to the third section from the left in Figure 2. $C$ (Female) and $D$ (Male) are from a more posterior level corresponding to the seventh section from the left in Figure 2. Abbreviations: $A C$, anterior commissure; $A H$, anterior hypothalamic nucleus; $M P N$, medial preoptic nucleus; $M P N a$, anterior compact subnucleus; $M P N c$, central compact subnucleus; $M P N p$, principal portion of medial preoptic nucleus; $O C$, optic chiasm; $L T$, lamina terminalis; $P e p$, preventricular periventricular nucleus; $S D N$, sexually dimorphic nucleus; $S O N$, supraoptic nucleus.

\section{Materials and Methods}

Adult albino guinea pigs of Hartley Stock, bred and reared in our animal facility, were used in this study. Breeding females were placed in the home cage of a stud male on the evening following the vaginal opening of each estrous cycle and removed the following morning, at which time a vaginal smear was taken by lavage. Pregnancies were timed, with the day of a sperm-positive smear designated as day 0 . The progeny were divided among the following 7 treatment groups:

Group 1. Gonadally intact males and females that received no treatment. This group was for assessment of the dimorphisms in normal adult animals.

Group 2. Neonatally gonadectomized males and females. This group was for assessment of the postnatal hormonal dependence of the dimorphisms. Neonatal gonadectomies were done $1-2$ weeks after birth since plasma testosterone in guinea pigs is nondetectable or present only in trace amounts prior to $15 \mathrm{~d}$ of age (Resko, 1970).

Group 3. Females that were gonadectomized neonatally and injected with $0.1 \mathrm{mg}$ testosterone propionate (TP) per day for $30 \mathrm{~d}$ immediately prior to sacrifice in adulthood. This postnatal TP treatment has been reported to significantly increase mounting frequencies in gonadectomized female guinea pigs (Groldfoot and Van Der Werf Ten Bosch, 1975) and was given for assessment of the effects of postnatal androgen exposure on the dimorphisms. The females that received this treatment are designated as postnatal TP-fermales.

Group 4. Vehicle control males and females. The mothers of these animals received corn oil $(0.1 \mathrm{ml})$ injections on gestational days 28-65.

Group 5 (E28-65 females). Females exposed to TP on embryonic (E) days $28-65$ by injecting their mothers with the hormone on the corresponding days of pregnancy.

Group 6 (E28-37 females). Females whose mothers received TP on gestational days $28-37$. 


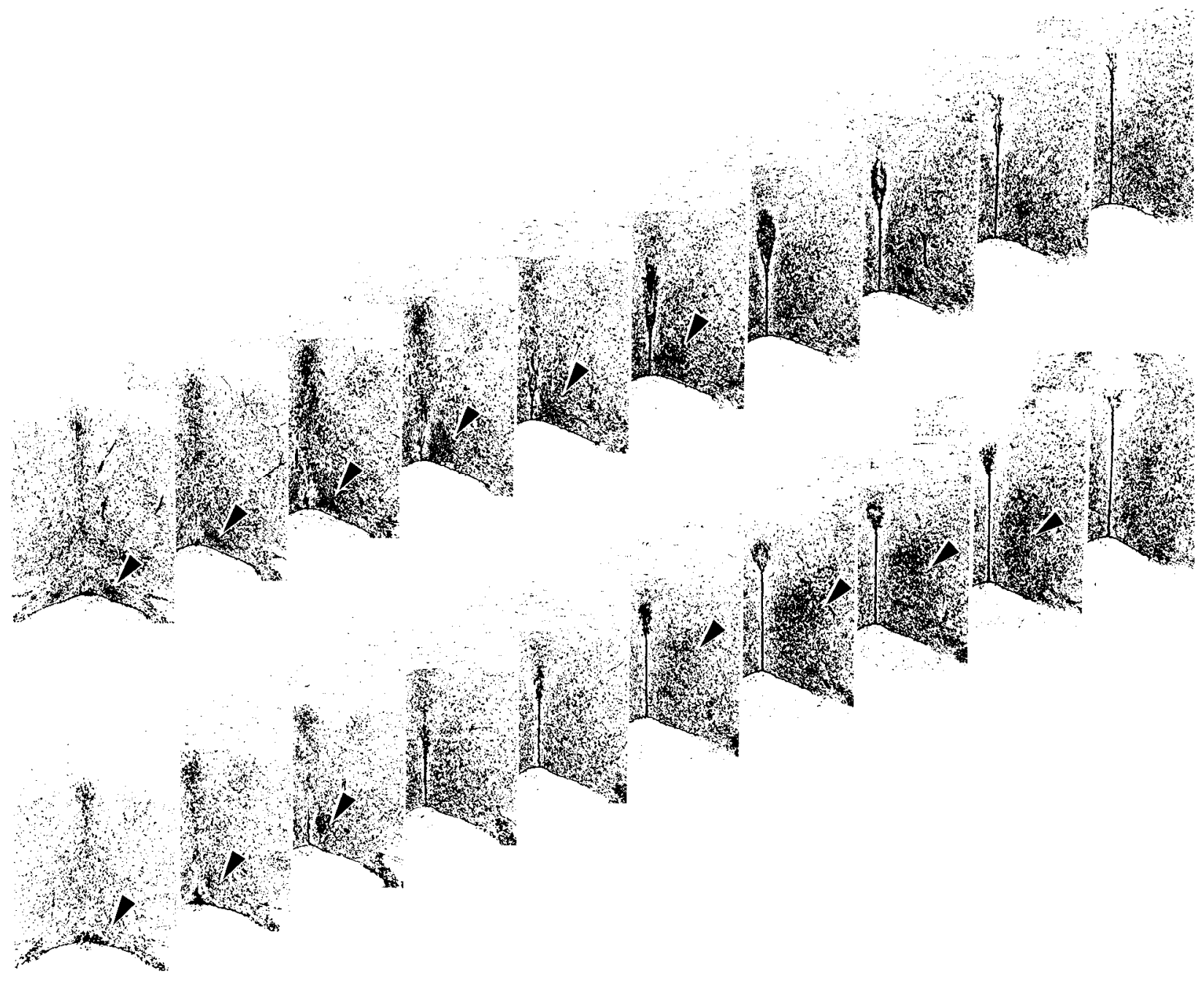

Figure 2. Alternate 30- $\mu$ m-thick sections through the MPN of a vehicle control female (top) and male (bottom) guinea pig. The most rostral section is at left. The arrows indicate the position of MPNa anteroventrally and MPNc more posterodorsally. An MPNc was not seen in this female. The MPNa volumes of these specimens are $20.72 \mathrm{~mm}^{3} \times 10^{-3}$ (female) and $4.35 \mathrm{~mm}^{3} \times 10^{-3}$ (male). The volume of MPNc in the male is 24.10 $\mathrm{mm}^{3} \times 10^{-3}$. Within the MPN, cells appear to be concentrated more anteroventromedially in the female than in the male.

Group 7 (E38-65 females). Females whose mothers received TP on gestational days $38-65$.

In each prenatal treatment regimen, the pregnant animals received 5 $\mathrm{mg} \mathrm{TP} / \mathrm{d}$ for the first $10 \mathrm{~d}$ of treatment and $1 \mathrm{mg}$ on the remaining days. The TP in each injection was dissolved in $0.1 \mathrm{ml}$ corn oil. Groups 57 were designed to assess the prenatal androgen dependence of the sexual dimorphisms following TP exposure according to one of 3 different treatment regimens, each of which was anticipated to have different functional consequences on the basis of previously published reports (Goy et al., 1964; Brown-Grant and Sherwood, 1971). The animals in groups 4-7 were gonadectomized at 4-6 months of age and were thereafter, until the time of sacrifice, given estradiol benzoate (EB) or sequential EB and progesterone injections biweekly in order to test the effects of these hormones on reproductive behaviors and luteinizing hormone (T.H) release. The prenatal vehicle control group (group 4) was therefore required in the event that postnatal administration of EB and progesterone influenced the dimorphisms. As this was not the case (see Results), data from groups 1,2 , and $\mathbf{4}$ were pooled to provide male and female controls with which to compare data from the prenatally androgenized females. The behavioral and LH data will be reported separately. The animals in groups 4-7 were sacrificed 2 weeks after their last postnatal hormone injection (approximately 3 months after gonadectomy).

Histology. All subjects were sacriticed at 7-9 months of age by an overdose of sodium pentobarbital. The brains were removed and sus- pended in $10 \%$ neutral formalin for 3 weeks. After fixation, the brains were weighed, dehydrated in graded alcohols, embedded in celloidin, sectioned serially at $30 \mu \mathrm{m}$ in a coronal plane approximating that of Paxinos and Watson (1986) and stained with thionin. Slides were coded so the sex and treatment of the preparations were not known to the investigators.

Quantitative cytoarchitectonic measurements. The brains of 50 animals were analyzed in this study: 5 brains of each sex from groups 1 , 2 , and 4 ; and 5 female brains only from groups $3,5-7$. Serial sections through the medial preoptic area were examined with a Leitz microscope fitted with a $2.5 \times$ objective and a drawing tube attachment with a $10 \times$ ocular. With the aid of the drawing tube, the boundaries of the MPNa and the MPNc were traced onto paper at a magnification of $60 \times$. Both nuclei were traced bilaterally by each of the investigators, who worked independently. The nuclear boundaries were then relayed from these tracings to a Harris (Datacraft) 6024 computer by the use of a TYLOS Cybergraphic Tablet. A package of computer programs, Cell Analysis Package (CAP), developed at our computer facility was then used to calculate the volumes of MPNa and MPNc. Neither investigator's measurements demonstrated a trend suggesting a left-right assymetry in the volume of either nucleus. Since the left and right volumes of each nucleus were virtually identical in all animals, the left and right volume measurements of each nucleus were averaged for each subject, and all analyses were carried out on these averages.

Statistical analyses. Statistical analyses were carried out with the 


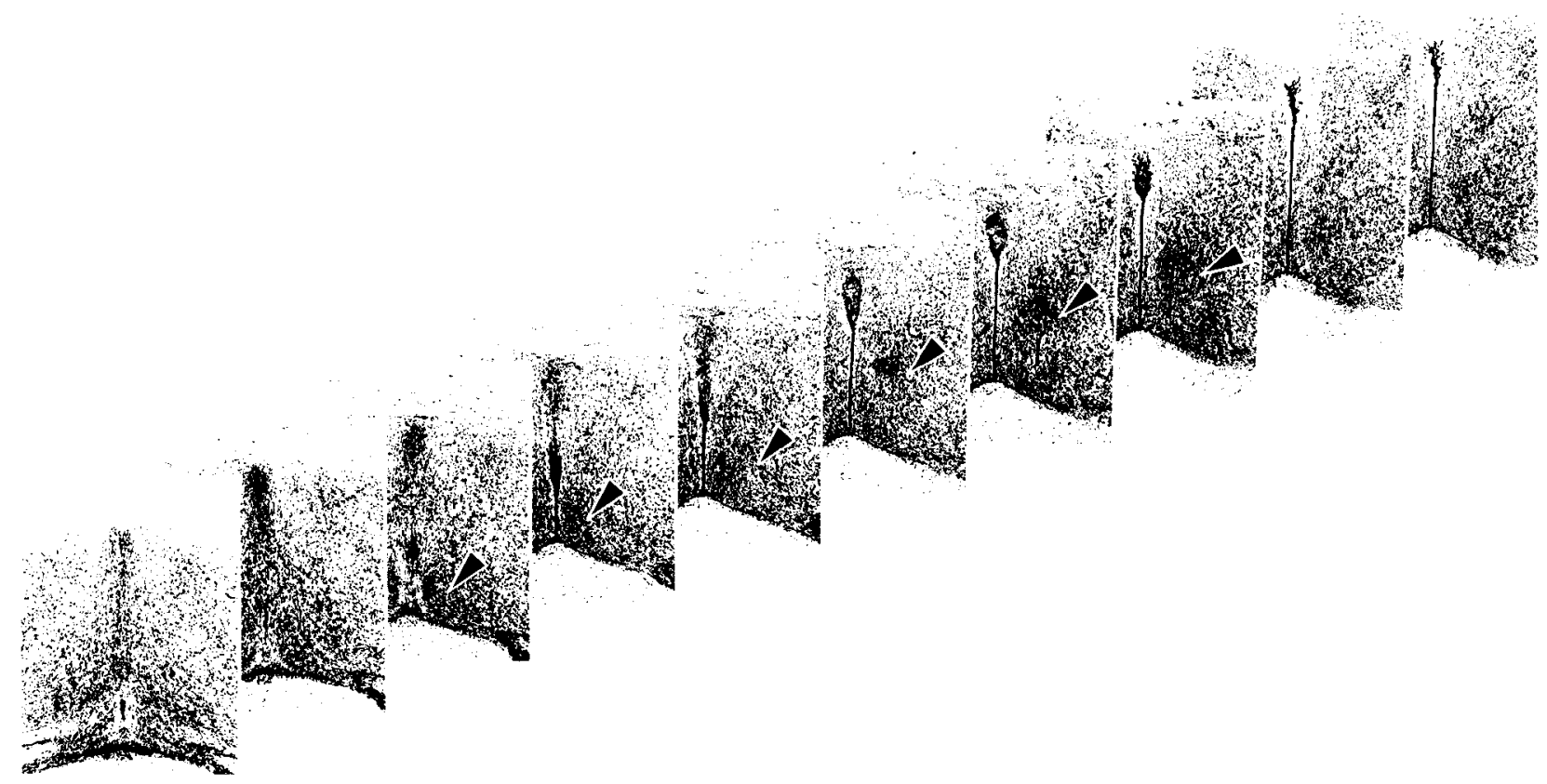

Figure 3. Alternate 30- $\mu \mathrm{m}$-thick sections through the MPN of a female guinea pig that received TP on gestational days $28-65$. The arrows indicate the position of MPNa anteroventrally and MPNc posterodorsally. The volumes of MPNa and MPNc are 7.00 and $19.00 \mathrm{~mm}^{3} \times 10^{-3}$, respectively.

Minitab (Ryan et al., 1976) statistical package and involved 1- and 2 -way (sex $\times$ treatment) analysis of variance for overall comparisons and $t$ tests for planned intergroup comparisons. The exact probability levels associated with each $t$ test are reported as calculated by Minitab.

Six separate statistical analyses were done: The measurements made by each investigator were analyzed independently and again after combining by averaging; these analyses were then repeated after dividing each volume measurement by the weight of the corresponding brain sincc analysis of variancc indicatcd significant diffcrences in brain wcight among groups $\left(F_{9,40}=2.30, p<0.05\right)$. As discussed under Results, independent analysis of the measurements made by the 2 investigators led to a different conclusion regarding the significance of only one comparison, and this was also the only comparison to be affected by the compensation for differences in brain weight. Unless specifically stated otherwise, all values in the text refer to comparisons involving the averaged volume measurements by the 2 investigators. Data are reported as means \pm SEM.

\section{Results}

MPNa was found to occupy an approximately 2-fold greater volume in normal gonadally intact females than in normal gonadally intact males $(p=0.009)$, while MPNc was found to be approximately 10 -fold larger in normal gonadally intact males than in normal gonadally intact females $(p=0.004)$. Neither the postnatal hormonal manipulations employed in this study nor TP exposure of females on E38-65 significantly altered the sexually dimorphic nuclei. In contrast, MPNa volume was significantly decreased and MPNc volume significantly increased in females exposed to TP on E28-65 or E28-37. The medial preoptic sexual dimorphisms of vehicle control male and female guinea pigs and of a female exposed to TP on E28-65 are shown in Figures 2 and 3. All data are summarized in Tables 1 and 2.

\section{The anterior compact subnucleus (MPNa)}

Two-way (sex $\times$ treatment) analysis of variance carried out over groups 1,2 , and 4 (the postnatal treatment groups that contained both sexes) revealed a significant effect of $\operatorname{sex}\left(F_{1,24}=45.00, p<\right.$ $0.001)$ but no significant postnatal treatment effect and no sex $x$ treatment interaction. The volume occupied by MPNa in the postnatal TP females did not differ significantly from that of the normal untreated adult females but was significantly larger than that of the neonatally gonadectomized adult males ( $p-$ 0.021 ). Thus, none of the postnatal hormonal manipulations significantly affected MPNa volume (Table 1, Fig. 4).

For comparisons involving prenatally androgenized females, data from the males in groups 1,2 , and 4 were pooled to serve as the control male reference, and data from the same female groups were pooled to provide the control female reference. MPNa was significantly larger in pooled control females than in pooled control males $(p=0.000)$. The volume of MPNa in E28-65 females and E28-37 females was significantly reduced compared to pooled female controls $(p=0.003$ and 0.000 , respectively) but did not differ significantly from pooled male controls. The volume of MPNa did not differ significantly between E28-65 and E28-37 females or between E38-65 females and pooled female controls. The average MPNa volume in E3865 females was approximately twice the average volume of E2865 and E28-37 females; however, these differences did not reach statistical significance ( $p=0.14$ and $p=0.091$, respectively). When analyzed independently, the measurements by the 2 investigators led to different conclusions regarding the significance of the difference in MPNa volume between pooled control males and E38-65 females, and these conclusions were not altered by correcting measurements for brain weight. When the measurements of the 2 investigators were combined by averaging and divided by brain weight, the difference in MPNa volume between these groups was significant (Table 2). The effects of the prenatal androgen treatments on the female MPNa are illustrated in Figure 5.

\section{The central compact subnucleus (MPNc)}

The sexual dimorphism in the MPNc, like that in MPNa, was found to depend upon prenatal rather than postnatal hormonal exposure. The same conclusions were reached in all comparisons involving the MPNc, regardless of whether the measurements 


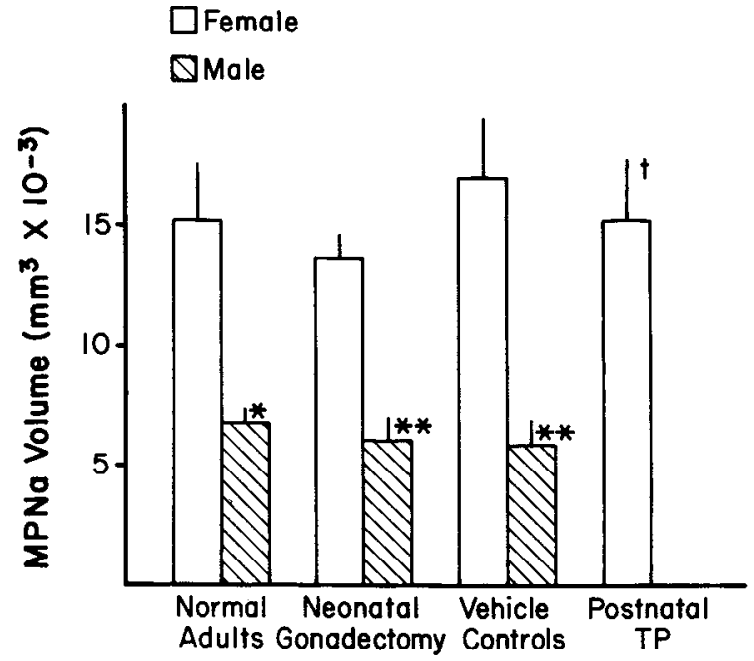

Figure 4. Persistence of the sexual dimorphism in MPNa volume despite postnatal hormonal manipulations. The vehicle control animals received vehicle injections prenatally, and the same postnatal $\mathrm{EB}$ and progesterone treatment regimen as the prenatally androgenized animals. ${ }^{*} p<0.05 ; * * p<0.01$. Significantly less than corresponding female group. † Significantly different from normal male control, $p<$ 0.05 .

made by the 2 investigators were averaged or analyzed independently and of whether compensation was made for differences in brain weight. Two-way (sex $\times$ treatment) analysis of variance carried out on MPNc volume over groups 1,2 , and 4 revealed a significant effect of $\operatorname{sex}\left(F_{1,24}=117.24, p<0.001\right)$ but no significant effect of postnatal treatment and no sex $\times$ treatment interaction (Table 1). The MPNc volume of postnatal TP females was significantly smaller than that of neonatally gonadectomized control males $(p=0.002)$ but not significantly different from that of normal untreated adult females. Thus, the sexual dimorphism in the volume of the MPNc was not significantly influenced by the postnatal hormonal manipulations employed in this study. Of the 20 females examined that had not received prenatal TP, an MPNc was found in only 9. The in-

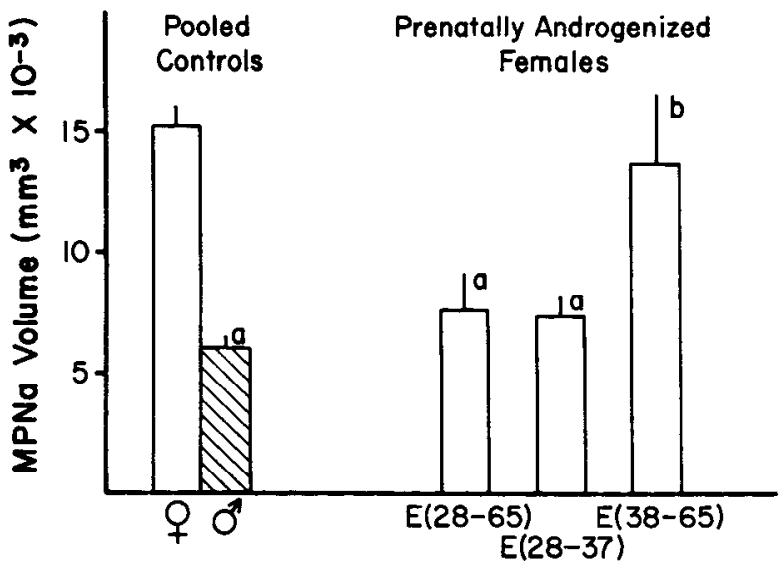

Figure 5. Effect of prenatal TP treatments on MPNa volume in genotypic females. ( $N=15$ for each of the control groups and 5 for each of the androgenized groups.) a Significantly different from control females $(p<0.01)$. ${ }^{b}$ Difference from control males approaches significance $(p=0.08)$. This difference became statistically significant $(p=$ 0.04 ) after dividing volumes by brain weights (see Table 2 ).

cidence of the MPNc in nonandrogenized female brains is considered in the Discussion. The average volume of the MPNc in those females in which it was identified was $(2.06 \pm 0.30 \times$ $\left.10^{-3} \mathrm{~mm}^{3}\right)$ and was significantly $(p=0.000)$ different from the average MPNc volume in pooled male controls $(20.56 \pm 1.68 \times$ $10^{-3} \mathrm{~mm}^{3}$ ). The persistence of the sexual dimorphism in the MPNc despite postnatal hormonal manipulations is illustrated in Figure 6.

For comparisons involving prenatally androgenized females, the data from groups 1,2 , and 4 were pooled to provide male and female controls (Table 2). The volume of the MPNc was significantly larger in E28-65 females and E28-37 females than in pooled control females ( $p=0.013$ and 0.001 , respectively). The MPNc volume of E38-65 females did not differ significantly from that of pooled control females. E28-37 and E38-65 females had significantly smaller MPNc volumes than the pooled control males ( $p=0.013$ and 0.000 , respectively). The MPNc

Table 1. Summary of experiments involving postnatal hormonal manipulations taking group differences in brain weight into account

\begin{tabular}{|c|c|c|c|c|c|c|}
\hline Group & $N$ & $\begin{array}{l}\text { Brain weight } \\
\text { (gm) }\end{array}$ & $\begin{array}{l}\text { MPNa volume } \\
\left(\mathrm{mm}^{3} \times 10^{-3}\right)\end{array}$ & $\begin{array}{l}\text { MPNa } \\
\text { volume/ } \\
\text { brain weight }\end{array}$ & $\begin{array}{l}\text { MPNc volume } \\
\left(\mathrm{mm}^{3} \times 10^{-3}\right)\end{array}$ & $\begin{array}{l}\text { MPNc } \\
\text { volume/ } \\
\text { brain weight }\end{array}$ \\
\hline \multicolumn{7}{|c|}{ Normal adult } \\
\hline Female & 5 & $4.30 \pm 0.18$ & $15.27 \pm 2.46^{a}$ & $3.59 \pm 0.61^{a}$ & $0.93 \pm 0.57^{b}$ & $0.20 \pm 0.12^{b}$ \\
\hline Male & 5 & $4.66 \pm 0.14$ & $6.89 \pm 0.62$ & $1.47 \pm 0.10$ & $22.42 \pm 3.55$ & $4.76 \pm 6.10$ \\
\hline \multicolumn{7}{|c|}{ Neonatally gonadectomized } \\
\hline Female & 5 & $4.49 \pm 0.16$ & $13.68 \pm 1.12^{b}$ & $3.04 \pm 0.17^{b}$ & $1.26 \pm 0.54^{b}$ & $0.27 \pm 0.12^{\circ}$ \\
\hline Male & 5 & $4.65 \pm 0.19$ & $6.12 \pm 0.95$ & $1.32 \pm 0.20$ & $19.13 \pm 2.90$ & $4.05 \pm 0.48$ \\
\hline \multicolumn{7}{|c|}{ Vehicle control } \\
\hline Female & 5 & $4.40 \pm 0.06$ & $16.95 \pm 2.45^{b}$ & $3.84 \pm 0.55^{b}$ & $1.02 \pm 0.65^{b}$ & $0.23 \pm 0.15^{b}$ \\
\hline Male & 5 & $4.38 \pm 0.23$ & $5.99 \pm 1.25$ & $1.38 \pm 0.29$ & $20.14 \pm 2.67$ & $4.58 \pm 0.74$ \\
\hline \multicolumn{7}{|c|}{ Postnatal TP } \\
\hline Female $^{c}$ & 5 & $4.07 \pm 1.12^{a}$ & $14.76 \pm 2.70^{a}$ & $3.58 \pm 0.58^{a}$ & $0.50 \pm 0.50^{b}$ & $0.12 \pm 0.12^{b}$ \\
\hline
\end{tabular}

Data are expressed as means \pm SEM.

${ }^{a} p<0.05$.

${ }^{i} p<0.01$. Significantly different from corresponding male group.

$c$ Neonatally gonadectomized males served as controls for these comparisons. 


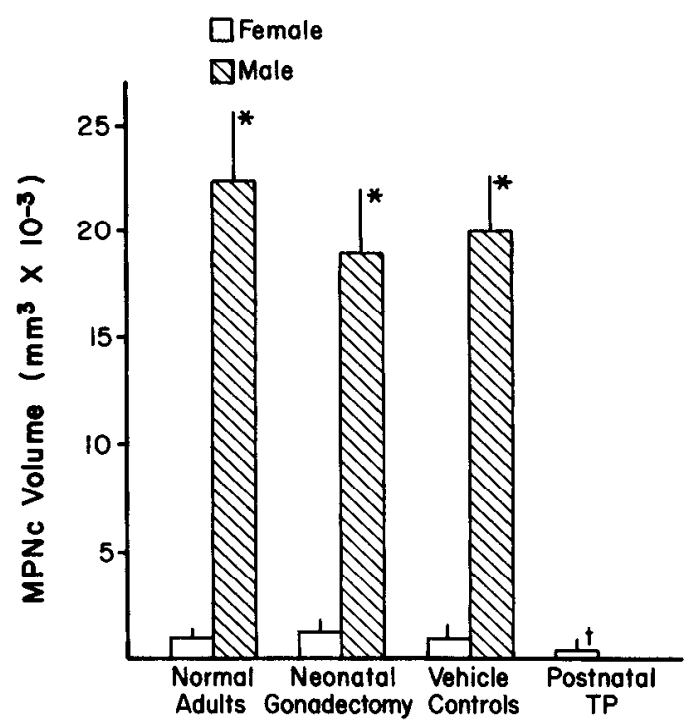

Figure 6. Persistence of sex difference in MPNc volume despite postnatal hormonal manipulations. The vehicle control animals received vehicle injections prenatally, and the same postnatal EB and progesterone treatment regimen as the prenatally androgenized animals. * Significantly different from corresponding female group $(p<0.01)$. $\dagger$ Significantly different from normal male controls $(p<0.01)$

volume in E28-65 females, however, did not differ significantly from that of pooled control males. The effects of the prenatal TP treatments on the MPNc volume of genotypic female guinea pigs are illustrated graphically in Figure 7.

\section{Discussion}

Three conclusions can be drawn from the data presented here: (1) The hypothalamic sexual dimorphisms previously described qualitatively (Bleier et al., 1982) can be demonstrated quantitatively; (2) these sex differences have little, if any, dependence upon postnatal hormonal exposure; and (3) the sex differences have a prenatal androgen dependence. Each of these conclusions will be addressed in turn.

Previously we reported that MPNa appeared to be more extensive in the female than in the male guinea pig (Bleier et al., 1982). This study confirms that report, finding the female's nucleus to be approximately twice as large as the male's. Our previous report described a cell group (here called the MPNc) corresponding to the SDN-POA of the rat in the male but not the female guinea pig. In females the entire medial region at the

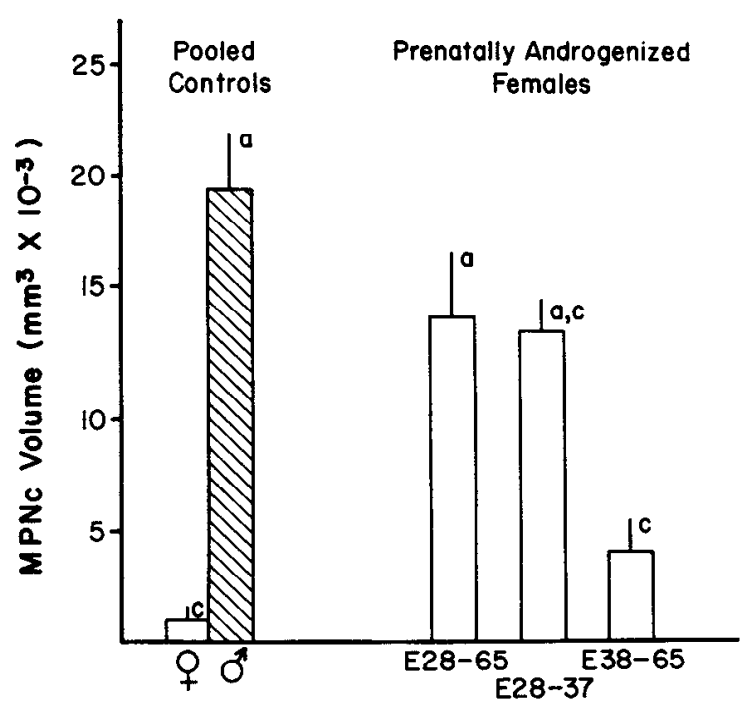

Figure 7. Effect of prenatal TP treatments on MPNc volume in genotypic females. ( $N=15$ for each of the control groups and 5 for each of the androgenized groups. ) a Significantly different from female control $(p \leq 0.01) .{ }^{\circ}$ Significantly different from male control $(p \leq 0.01)$.

level at which the MPNc was seen in the male was described as being uniformly cellular, lacking the contrast in cell density that gave definition to the MPNc in the male. In the present study; however, an MPNc was found in the hypothalamus of 9 out of 20 females that had not been treated with androgens prenatally. A recent report from another laboratory (Hines et al., 1985) also distinguished this cell group in the female guinea pig but apparently in all 16 females examined. In Nissl preparations MPNc is defined solely on the basis of its increased density relative to its surround. Thus, the discrepancy in the incidence of the MPNc in female guinea pigs is most likely attributable to differences between laboratories in the precise tilt of the frontal planes of sectioning employed. Hines et al. used the plane of de Groot (1959), whereas the plane used in this study and that of Bleier et al. (1982) approximates the plane of Paxinos and Watson (1986), which differs from the de Groot plane by approximately $30^{\circ}$. When the de Groot plane transects the MPNc dorsally, it passes ventrally through an area of relative cell sparcity (the anterior hypothalamus overlying the suprachiasmatic nucleus), enhancing the contrast in density that defines the MPNc more dorsally. In contrast, when our plane of sectioning passes through the region of the MPNc dorsally, the ventral portion of the plane lies within the densely cellular MPNp.

Table 2. Summary of experiments involving prenatal hormonal manipulations taking group differences in brain weight into account

\begin{tabular}{|c|c|c|c|c|c|c|}
\hline Group & $N$ & $\begin{array}{l}\text { Brain weight } \\
\text { (gm) }\end{array}$ & $\begin{array}{l}\text { MPNa volume } \\
\left(\mathrm{mm}^{3} \times 10^{-3}\right)\end{array}$ & $\begin{array}{l}\text { MPNa volume/ } \\
\text { brain weight }\end{array}$ & $\begin{array}{l}\text { MPNc volume } \\
\left(\mathrm{mm}^{3} \times 10^{-3}\right)\end{array}$ & $\begin{array}{l}\text { MPNc volume/ } \\
\text { brain weight }\end{array}$ \\
\hline Pooled control female & 15 & $4.40 \pm 0.08$ & $15.30 \pm 1.18^{c}$ & $3.49 \pm 0.03^{c}$ & $1.07 \pm 0.39^{c}$ & $0.22 \pm 0.09^{c}$ \\
\hline Pooled control male & 15 & $4.56 \pm 0.11$ & $6.33 \pm 0.53^{a}$ & $1.39 \pm 0.11^{a}$ & $20.56 \pm 1.68^{a}$ & $4.50 \pm 0.34^{a}$ \\
\hline$E(28-65)$ female & 5 & $4.33 \pm 0.24$ & $7.63 \pm 1.50^{a}$ & $1.81 \pm 3.60^{\circ}$ & $13.61 \pm 2.88^{a}$ & $3.16 \pm 0.64^{a}$ \\
\hline$E(28-37)$ female & 5 & $3.92 \pm 0.09^{a, c}$ & $6.85 \pm 1.03^{a}$ & $1.74 \pm 0.25^{a}$ & $12.99 \pm 1.37^{a, c}$ & $3.34 \pm 0.39^{a, b}$ \\
\hline$E(38-65)$ female & 5 & $4.06 \pm 0.14^{b}$ & $12.89 \pm 2.70$ & $3.13 \pm 0.58^{b}$ & $4.12 \pm 1.26^{c}$ & $1.05 \pm 0.34^{c}$ \\
\hline
\end{tabular}

Data are expressed as means \pm SEM.

a Significantly different from pooled control female, $p<0.01$

b Significantly different from poolcd control malc, $p<0.05$.

Significantly different from pooled control male, $p<0.01$. 
Thus, in the female guinea pig an ovoid configuration of increased density that resembles the MPNc of the male is more readily identifiable in the de Groot plane than in the plane of this study. In males, the MPNc is more robust and is easily identifiable in either plane.

Neither the volume of the MPNa nor that of the MPNc was significantly affected by any of the postnatal hormonal manipulations employed in this study. Hines et al. (1985) also failed to detect significant postnatal hormonal effects on cytoarchitectonic hypothalamic sexual dimorphisms in the guinea pig. TP exposure on E28-65 or E28-37 but not E38-65 significantly decreased MPNa volume and increased MPNc volume in female guinea pigs. Although statistically significant differences between E28-65 females and control males were not found, inspection of the photomicrographs of Figures 2 and 3 suggests that the hypothalamic cytoarchitecture of the E28-65 females was qualitatively different from that of control males. Furthermore, inspection of our data suggests that the failure to find statistically significant differences between these 2 groups may be a Type $I I$ statistical error. Appropriately timed hormonal administration has been reported to completely masculinize the SDN-POA of female rats (Döhler et al., 1984). Thus, the most likely explanation for our failure to achieve complete cytoarchitectonic masculinization is that our earliest TP treatments were initiated on E28, while the fetal guinea pig testes begin steroid synthesis at approximately E25. Sex differences in plasma testosterone levels are evident on E27, the earliest day investigated in this regard (Buhl et al., 1979; Rigaudiere, 1979). E28 was chosen as the starting day for our treatments in order to test the correlation between the functional and cytoarchitectonic changes induced by androgens since E28-37 has been reported to include the periods of maximal susceptibility to the organizational actions of androgens on behavioral and endocrinological reproductive functions regulated by the hypothalamus (Goy et al., 1964; Brown-Grant and Sherwood, 1971; Goy and McEwen, 1980).

All 3 prenatal TP treatment regimens administered to females in this study significantly increased mounting frequencies and suppressed lordosis, ovulation, and the positive-feedback effects of estrogen and progesterone on LH release (Byne, 1985). In contrast, TP administration on E28-65 or E28-37 but not E3865 had significant masculinizing effects on hypothalamic cytoarchitecture and external genitalia. Thus, the period during which hypothalamic cytoarchitecture is organized by androgens corresponds closely to the period of sexual differentiation of the external genitalia and appears to be more restricted than the period during which hypothalamic functions are susceptible to androgenic organizational influences. Our results, therefore, suggest that the sex differences in hypothalamic cytoarchitecture alone are not sufficient to account for sex differences in hypothalamic functions. Sex differences in hypothalamic neuropil, hormone receptor mechanisms, and neurotransmitter systems are well documented in rodents, and we suggest that androgens may exert organizational influences on any or all of these substrates after cytoarchitectonic patterns have been determined (Raisman and Field, 1973; Cidlowski and Muldoon, 1976; Dyer et al., 1976; Greenough et al., 1977; Whalen and Olsen, 1978; Blaustein et al., 1980; Toran-Allerand et al., 1980; Watson et al., 1983; Commins and Yahr, 1984b, 1985; Hammer, 1984; Simerly et al., 1984, 1985a, b). In the rat, androgens exert their organizational effects upon hypothalamic structure after they are converted to estrogens through the process of aromatization (for review, see Goy and McEwen, 1980). Aromatization may also be required in the sexual differentiation of hypothalamic structure in the guinea pig since TP, an aromatizable androgen, was used in the present studies, and since prenatal exposure to diethylstilbestrol masculinizes the cyloarchitecture of the MPNc of female guinea pigs (M. Hines, personal communication).

In our earlier report, we described a sexually dimorphic nuclear complex that includes not only those subnuclei of the MPN described here, but also the anterior periventricular nucleus (Pea) and the bed nucleus of the stria terminalis (BNST). A volumetric sex difference in a component of the BNST has recently been described in the guinea pig by Hines et al. (1985). The sexually dimorphic nuclear complex forms a cellular continuum extending through the medial preoptic area and dorsally into the BNST. It is characterized and distinguished from its surround not only by its greater cell density, but also by its density of cells with steroid hormone receptors (Sar and Stumpf, 1975a, b; Sheridan et al., 1975; Stumpf et al., 1975; Warembourg, 1977) and by its patterns of neurogenesis. In the guinea pig the sexually dimorphic nuclei are the last nuclei in the medial preoptic region in which neuronal proliferation ceases, and they are the only nuclei in the region in which significant neuronal proliferation occurs after the onset of fetal gonadal activity (Byne et al., 1987). Thus, from the moment of their production, the developmental course of neurons destined for the sexually dimorphic nuclei may be influenced by sex differences in the levels of gonadal steroids. Connectional evidence reviewed by Hines et al. (1985) suggests that the sexually dimorphic nuclear complex of the MPA may be part of a larger functional and interconnected neural network involving portions of the ventromedial hypothalamus and amygdala that are also characterized by sexual dimorphism and a high density of steroid hormone receptors.

Various lesion, hormonal implantation, and electrical stimulation studies have consistently implicated the MPA in the regulation of a number of sexually differentiated functions in rodents, including the expression of stereotypic mating behaviors and the release of gonadotropins (for reviews, see Goy and McFwen, 1980; Terasawa and Davis, 1983). In the present study, however, there was only a poor association between the structural and functional effects of TP on the hypothalamus. Furthermore, bilateral destruction of the MPNc has not been observed to disrupt any sexually differentiated function (Arendash and Gorski, 1983). There is, however, evidence that MPNa has a role in the initiation of ovulation in the rat (for review, see Terasawa and Davis, 1983) and guinea pig (Terasawa et al., 1982). Further, severalfold more dopaminergic cell bodies and fibers have been found in the MPNa of female as compared to male rats, suggesting that dopamine may participate in the regulation of gonadotropin secretion at the level of this nucleus (Simerly et al., 1985a).

In light of the temptation to extrapolate findings in rodents to other species, it should be noted that the hypothalami of rodents and primates do not appear to be similarly sexually differentiated (Gooren, 1986a, b; Norman and Spies, 1986). Although the functional significance of hypothalamic sexual dimorphisms in rodents cannot be automatically extrapolated to other species, such sex differences nevertheless provide powerful model systems for the study of hormonal influences on neural development, as well as for the study of the relationship between 
neural structure and neural responses to complex environmental and hormonal stimuli.

\section{References}

Arendash, G. W., and R. A. Gorski (1983) Effects of discrete lesions of the sexually dimorphic nucleus of the preoptic area or other medial preoptic regions on the sexual behavior of male rats. Brain Res. Bull. 10: $147-154$.

Blaustein, J. D., H. I. Ryer, and H. H. Feder (1980) A sex difference in the progestin receptor system of guinea pig brain. Neuroendocrinology 31: 403-409.

Bleier, R., W. Byne, and I. Siggelkow (1982) Cytoarchitectonic sexual dimorphisms of the medial preoptic and anterior hypothalamic areas in the guinea pig, ral, hamster and mouse. J. Comp. Neurol. 212: 118-130.

Brown-Grant, K., and M. R. Sherwood (1971) The "early androgen syndrome" in the guinea pig. J. Endocrinol. 49: 277-291.

Buhl, A. E., L. M. Pasztor, and J. A. Resko (1979) Sex steroids in guinea pig fetuses after sexual differentiation of the gonads. Biol. Reprod. 21: 905-909.

Butler, J. E., and B. T. Donovan (1970) The effect of surgical isolation of the hypothalamus upon reproductive function in the female guinea pig. J. Endocrinol. 50: 507-514.

Byne, W. (1985) Cytoarchitectonic sexual dimorphisms in the guinea pig: Morphometric, functional and ontogenetic studies. Ph.D. thesis, University of Wisconsin, Madison.

Byne, W., J. T. Warren, and I. Siggelkow (1987) Medial preoptic sexual dimorphisms in the guinea pig. II. An investigation of medial preoptic neurogenesis. J. Neurosci. 7: 2697-2702.

Cidlowski, J. A., and T. G. Muldoon (1976) Sex related differences in the regulation of cytoplasmic estrogen receptor levels in responsive tissues of the rat. Endocrinology 98: 833-841.

Commins, D., and P. Yahr (1984a) Adult testosterone levels influence and morphology of a sexually dimorphic area in the Mongolian gerbil brain. J. Comp. Neurol. 224: 132-140.

Commins, D., and P. Yahr (1984h) Acetylcholinesterase activity in the sexually dimorphic area of the gerbil brain: Sex differences and influences of adult gonadal steroids. J. Comp. Neurol. 224: 123-131.

Commins, D., and P. Yahr (1985) Autoradiographic localization of estrogen and androgen receptors in the sexually dimorphic area and other regions of the gerbil brain. J. Comp. Neurol. 231: 473-489.

de Groot, J. (1959) The rat forebrain in stereotaxic coordinates. J. Comp. Neurol. 113: 389-400.

Döhler, K.-D., A. Coquelin, F. Davis, M. Hines, J. E. Shryne, and R. A. Gorski (1984) Pre- and postnatal influence of testosterone propionate and diethylstilbestrol on differentiation of the sexually dimorphic nucleus of the preoptic area in male and female rats. Brain Res. 302: 291-295.

Dyer, R. G., N. K. McLeod, and F. Ellendorf (1976) Electrophysiological evidence for sexual dimorphism and synaptic convergence in the preoptic and anterior hypothalamic area of the rat. Proc. R. Soc. London [Biol.] 193: 421-440.

Goldfoot, D. A., and J. J. Van Der Werf Ten Bosch (1975) Mounting behavior of female guinea pigs after prenatal and adult administration of the propionates of testosterone, dihydrotestosterone, and androstanediol. Hormones Behav. 6: 139-148.

Gooren, L. (1986a) The neuroendocrine response of luteinizing hormone to estrogen administration in heterosexual, homosexual and transexual subjects. J. Clin. Endocrinol. Metab. 63: 583-588.

Gooren, L. (1986b) The neuroendocrine response of luteinizing hormone to estrogen administration in the human is not sex specific but dependent on the hormonal environment. J. Clin. Endocrinol. Metab. 63: 589-593.

Gorski, R. A., I. H. Gordon, J. E. Shryne, and A. M. Southam (1978) Evidence for a morphological sex difference within the medial preoptic area of the rat brain. Brain Res. 148: 333-346.

Gorski, R. A., R. E. Hanlan, C. D. Jacobson, and J. E. Shryne (1980) Evidence for the existence of a sexually dimorphic nucleus in the preoptic area of the rat. J. Comp. Neurol. 193: 529-539.

Goy, R. W., and B. S. McEwen (1980) Sexual Differentiation of the Brain, MIT Press, Cambridge, MA.

Goy, R. W., W. E. Bridson, and W. C. Young (1964) Period of maximal susceptibility of the prenatal female guinea pig to the masculinizing actions of testosterone propionate. J. Comp. Physiol. Psychol. 57: 166-174.

Greenough, W. T., C. S. Carter, C. Steerman, and T. J. deVoogd (1977) Sex differences in dendritic patterns in hamster preoptic area. Brain Res. 126: 63-72.

Gurdjian, E. S. (1927) The diencephalon of the albino rat. J. Comp. Neurol. 43: 1-114.

Hammer, R. P. (1984) The sexually dimorphic region of the preoptic area in rats contains denser opiate receptor binding sites in females. Brain Res. 308: 172-176.

Hines, M., F. C. Davis, A. Coquelin, R. W. Goy, and R. A. Gorski (1985) Sexually dimorphic regions in the medial preoptic area and the bed nucleus of the stria terminalis of the guinea pig brain. $\mathbf{J}$. Neurosci. 5: 40-47.

Konig, J. F. R., and R. A. Klippel (1963) The Rat Brain. A Stereotaxic Atlas of the Forebrain and Lower Parts of the Brian Stem, Williams \& Wilkins, Baltimore, MD.

Meyers, P. E., and J. H. Gordon (1982) Sexually dimorphic dendritic organization in the preoptic area of the neonatal rat. Soc. Neurosci. Abstr. 8: 637.

Norman, R. L., and H. G. Spies (1986) Cyclic ovarian function in a male macaque: Additional evidence for a lack of sexual differentiation in the physiological mechanisms that regulate the cyclic release of gonadotropins in primates. Endocrinology 118: 2608-2610.

Paxinos, G., and C. Watson (1986) The Rat Brain in Stereotaxic Coordinates, 2nd ed., Academic, Sydney.

Raisman, G., and P. M. Field (1973) Sexual dimorphism in the neuropil of the preoptic area of the rat and its dependence on neonatal androgen. Brain Res. 54: 1-29.

Resko, J. A. (1970) Androgens in systemic plasma of male guinea pigs during development and after castration in adulthood. Endocrinology 86: 1444-1447.

Rigaudiere, N. (1979) The androgens in the guinea pig fetus throughout the embryonic development. Acta Endocrinol. 92: 174-186.

Ryan, T. A., B. L. Joiner, and B. F. Ryan (1976) Minitab Student Handbook, Duxbury Press, North Scituate, MA

Sar, M., and W. E. Stumpf (1975a) Distribution of androgen concentrating neurons in rat brain. In Anatomical Neuroendocrinology, W. E. Stumpf and L. D. Grant, eds., pp. 120-133, Karger, New York.

Sar, M., and W. E. Stumpf (1975b) Cellular localization of progestin estrogen in guinea pig hypothalamus by autoradiography. In Anatomical Neuroendocrinology, W. E. Stumpf and L. D. Grant, eds., pp. 142-152, Karger, New York.

Sheridan, P. J., M. Sar, and W. E. Stumpf (1975) Estrogen and androgen distribution in the brain of neonatal rats. In Anatomical Neuroendocrinology, W. E. Stumpf and L. D. Grant, eds., pp. 134-141, Karger, New York.

Simerly, R. B. L., L. W. Swanson, and R. A. Gorski (1984) Demonstration of a sexual dimorphism in the distribution of serotonin immunoreactive fibers in the medial preoptic nucleus of the rat. J. Comp. Neurol. 225: 151-166.

Simerly, R. B. L., L. W. Swanson, and R. A. Gorski (1985a) The distribution of monoaminergic cells and fibers in a periventricular preoptic nucleus involved in the control of gonadotropin release: Immunohistochemical evidence for a dopaminergic sexual dimorphism. Brain Res. 330: 55-64.

Simerly, R. B. L., L. W. Swanson, R. J. Handa, and R. A. Gorski (1985b) Influence of perinatal androgen on the sexually dimorphic distribution of tyrosine hydroxylase-immunoreactive cells and fibers in the anteroventral periventricular nucleus of the rat. Neuroendocrinology 40: 501-510.

Stumpf, W. E., M. Sar, and D. A. Keffer (1975) Atlas of estrogen target cells in the rat brain. In Anatomical Neuroendocrinology, W. E. Stumpf and L. D. Grant, eds., pp. 104-119, Karger, New York.

Terasawa, E., and G. A. Davis (1983) The LHRH neuronal system in female rats: Relation to the medial preoptic nucleus. Endocrinol. Jpn. 30: 405-417.

Terasawa, E., and S. J. Wiegand (1977) Effects of hypothalamic deafferentation on ovulation and estrous cyclicity in the female guinea pig. Neuroendocrinology 26: 229-248.

Terasawa, E., J. A. Keller-Halbe, and S. J. Wiegand (1982) A role of the medial preoptic nucleus in regulation of cyclic gonadotropin release in the female guinea pig. Soc. Study Reprod. Abstr. 15: 49A.

Terasawa, E., R. R. Yeoman, and N. J. Schultz (1984) Factors influ- 
encing the progesterone-induced luteinizing hormone surge in rhesus monkeys: Diurnal influence and time interval after estrogen. Biol. Reprod. 31: 732-741.

Tobet, S. A., D. J. Zahniser, and M. J. Baum (1986) Differentiation in male ferrets of a sexually dimorphic nucleus of the preoptic/anterior hypothalamic area requires prenatal estrogen. Neuroendocrinology 44: 299-308.

Toran-Allerand, C. D., J. L. Gerlach, and B. S. McEwen (1980) Sex steroids and the development of the newborn mouse hypothalamus and preoptic area in vitro. Brain Res. 184: 517-522.

Vito, C. C., S. J. Wieland, and T. O. Fox (1979) Androgen receptors exist throughout the "critical period" of brain sexual differentiation. Nature 282: 308-310.

Warembourg, M. (1977) Radioautographic localization of estrogenconcentrating cells in the brain and pituitary of the guinea pig. Brain Res. 123: 357-362.

Watson, R. E., G. E. Hoffman, and S. J. Wiegand (1983) The medial preoptic nucleus: Peptidergic components. Soc. Neurosci. Abstr. 9: 454.

Whalen, R. E., and K. L. Olsen (1978) Chromatin binding of estradiol in the hypothalamus and cortex of male and female rats. Brain Res. 152: $121-131$. 